\title{
The prophylactic use of a proton pump inhibitor (PPI) in patients treated with clopidogrel and aspirin for an acute coronary syndrome or placement of a coronary stent reduces the rate of upper gastrointestinal bleeding with no apparent increase in cardiovascular events
}

\author{
Simona Sada $\cdot$ Giorgio Costantino
}

Received: 3 January 2011 / Accepted: 17 February 2011/Published online: 3 March 2011

(c) SIMI 2011

\section{Background}

In recent years, more and more patients have been treated with dual antiplatelet therapy (clopidogrel and aspirin), mainly for acute coronary syndrome or after the placement of a coronary stent [1]. While this therapy is efficacious in reducing cardiovascular events, there are some worries about its safety, in particular regarding the risk of upper gastrointestinal haemorrhage. In this regard, proton pump inhibitors (PPI)s reduce the risk of bleeding in patients treated with low dose aspirin or non-steroidal antinflammatory drugs (NSAIDs), and might be an attractive way to improve the safety of dual antiplatelet therapy [2]. Biological studies have shown a broad inter-individual variability of platelet response to clopidogrel, and more recently, a low response to clopidogrel has been associated with an increased risk of ischemic events [3]. Several mechanisms have been proposed to explain this variability of response, including genetic factors and interaction with other medications. Clopidogrel is a prodrug converted in the liver to its active form by cytochrome P450 isoenzymes, with the CYP450 isoform 2C19 playing a major role in this metabolism. There is evidence suggesting that various PPIs, and most consistently omeprazole [4] can inhibit CYP2C19, which alters the effectiveness of

Simona Sada and Giorgio Costantino on behalf of GrAM.

\section{S. Sada}

Divisione di Medicina Interna B, Azienda Ospedaliera di

Garbagnate, Milano, Italy

G. Costantino ( $\square)$

Medicina II, Ospedale L. Sacco, Università degli Studi di

Milano, Via GB grassi 74, 20157 Milano, Italy

e-mail: giorgic@libero.it clopidogrel, and potentially leads to an increased risk of adverse cardiovascular outcomes.

\section{Summary}

In the COGENT trial [5], 3,873 patients with an indication for a dual antiplatelet therapy were randomly assigned to omeprazole or placebo. The primary gastrointestinal (GI) end point was a composite of overt or occult bleeding, symptomatic gastroduodenal ulcers or erosions, obstruction or perforation. The primary cardiovascular end point was a composite of death from cardiovascular causes, nonfatal myocardial infarction, revascularization or stroke. Fiftyone patients presented the primary GI end point, with an event rate of $1.1 \%$ in the omeprazole group and $2.9 \%$ in the placebo group (hazard ratio $0.34,95 \%$ CI $0.18-0.63$; $P<0.001)$. The number of patients who would need to be treated for 6 months to prevent one occurrence of an event that was part of the primary GI end point is 55 .

On the side of safety, 109 patients presented a cardiovascular outcome, $4.9 \%$ with omeprazole and $5.7 \%$ with placebo (hazard ratio $0.99,95 \%$ CI $0.68-1.44 ; P=0.96$ ).

The authors conclude that among patients receiving aspirin plus clopidogrel, the prophylactic use of a PPI reduces the rate of upper gastrointestinal bleeding, but the observed results do not rule out a clinically meaningful difference in cardiovascular events.

\section{Strengths of the study}

- The GI end point is clinically relevant. While finding endoscopic alterations in patients assuming dual antiplatelet therapy (used in previous studies) may be 
irrelevant (as it is a surrogate end point), the end point chosen by the authors is important in daily practice;

- It is a well designed randomized controlled trial for the purpose of the analysis of the GI end point;

- Although the study is sponsored by industry, all the analysis and the data management have been independently performed by academic scientists.

\section{Weakness of the study}

- It is unusual to see two different primary end points in the same trial. Moreover, while the study is well designed and conducted for the GI primary end point, probably a different study design would have been more appropriate in order to investigate the cardiovascular end point. The absence of a significant difference between the two groups, as the authors state, cannot rule out a possible meaningful difference for the primary cardiovascular outcome. The broad CI of the cardiovascular end point is also in support of this assertion.

\section{Question marks}

It would be interesting to know if the aspirin dose (authors state that it is between 75 and $325 \mathrm{mg} /$ day) was equal in the omeprazole and control groups. Indeed this data was not reported in the article.

\section{Clinical bottom line}

Omeprazole reduces the risk of upper gastrointestinal bleeding in patients with dual antiplatelet therapy. Caution should be used in patients at high cardiovascular risk because the cardiovascular results of this trial are not conclusive.

Conflict of interest None.

\section{References}

1. Anderson JL, Adams CD, Antman EM et al (2007) ACC/AHA guidelines for the management of patients with unstable angina/ non-st-elevation myocardial infarction. JACC 50:1-157

2. Bhatt DL, Scheiman J, Abraham NS et al (2008) American College of Cardiology Foundation Task Force on Clinical Expert Consensus Documents ACCF/ACG/AHA expert consensus document on reducing the gastrointestinal risks of antiplatelet therapy and NSAID use: a report of the American College of Cardiology Foundation Task Force on Clinical Expert Consensus Documents. JACC 52:1502-1517

3. Matetzky S, Shenkman B, Guetta V et al (2004) Clopidogrel resistance is associated with increased risk of recurrent atherothrombotic events in patients with acute myocardial infarction. Circulation 109:3171-3175

4. Cuisset T, Frere C, Quilici J et al (2009) Comparison of omeprazole and pantoprazole influence on a high 150-mg clopidogrel maintenance dose the PACA (Proton Pump Inhibitors And Clopidogrel Association) prospective randomized study. JACC 54:1149-1153

5. Bhatt DL, Cryer BL, Contant CF et al (2010) Cogent investigators clopidogrel with or without omeprazole in coronary artery disease. N Engl J Med 363:1909-1917 